\title{
REVIEW
}

\section{Neurological emergencies: acute stroke}

\author{
Richard Davenport, Martin Dennis
}

\begin{abstract}
Stroke causes a vast amount of death and disability throughout the world, yet for many healthcare professionals it remains an area of therapeutic nihilism, and thus uninteresting. This negative perception is shared by the general public, who often have a poor understanding of the early symptoms and significance of a stroke. Yet within the past few years there have been many important developments in the approach to caring for stroke patients, for both the acute management and secondary prevention. After the completion of numerous clinical trials, there is now robust evidence to either support or discredit various interventions. Even more exciting is the prospect of yet more data becoming available in the near future, testing a whole array of treatments, as clinical interest in stroke expands exponentially. In this review an evidence based approach to the management of acute stroke within the first few days is presented, including ischaemic and haemorrhagic events, but not subarachnoid haemorrhage. It is explained why stroke is regarded as a medical emergency, and the importance of a rational, methodic approach to the initial assessment, which is the key to accurate diagnosis and subsequent management, is emphasised. The potential early problems associated with stroke are identified and specific interventions for different stroke types are discussed. The review ends with a brief discussion of the implications that the evolving treatments have for the organisation of modern stroke services. (F Neurol Neurosurg Psychiatry 2000;68:277-288)
\end{abstract}

Keywords: acute stroke

In the western world, stroke is the third commonest cause of death (after heart disease and all cancers), is probably the commonest cause of severe disability, ${ }^{12}$ and accounts for a large proportion of healthcare resources. Its impact on individual patients, their families, and society as a whole is immense. About 200 people per 100000 population will have a first ever stroke every year. ${ }^{3}$ Their mean age is about 72 years, and men and women are affected in roughly equal numbers. Despite the uncertainty over whether stroke incidence is rising, falling, or remaining static, ${ }^{34}$ the absolute number of patients is likely to increase, as incidence increases with age and most populations are aging.

Stroke has in the past held a low priority for many professional groups; in 1988 the King's Fund Forum concluded that hospital, primary care and community services for stroke in the United Kingdom were "haphazard, fragmented, and poorly tailored to the patients' needs". ${ }^{5}$ However, increasing awareness of the impact of stroke has led to it being identified as a priority for improving services and research. ${ }^{6}$ Numerous well conducted randomised trials and systematic reviews of medical and surgical treatments for acute stroke, as well as primary and secondary prevention strategies, mean that we are now considerably better equipped to know which treatments work, and which should be abandoned, and what sort of services we should be providing for our patients.

In this review, we will describe our approach to the management of acute stroke, focusing mainly on the first few days. Although the World Health Organisation (WHO) definition of stroke (see below) includes subarachnoid haemorrhage, we will not consider this distinct syndrome further, as it is covered in detail elsewhere in this series. Our approach is based on our interpretation of the available evidence, with particular emphasis on randomised controlled trials and systematic reviews, as we think that these provide the most reliable data on the risks and benefits of treatments. However, where such evidence is either absent or insufficient (and despite the many welcome advances, there is still much we do not know for sure), we will describe what we do in routine practice. We accept that other interpretations of the evidence are possible and are likely to be influenced by the context in which one works. For example, in the United Kingdom, unlike some other countries, our patients tend not to demand "treatment" (whether of proved benefit or not), "fee for service" is rare, and the resources available for health care are restricted so that only cost effective treatments will be advocated for widespread use. Lastly, although rehabilitation and secondary prevention are not the focus of this review, it is important to emphasise that for many patients, their management after the 
acute stage currently has the greater impact on their lives; with the advent of new acute strategies, this balance may change.

\section{Is acute stroke an emergency?}

Medical emergencies can be defined by certain criteria including rapidity of onset, poor prognosis, and requirement for prompt intervention. Although stroke has traditionally been treated as less of an emergency than, for instance, acute myocardial infarction or meningitis, we illustrate that this conservative approach is no longer tenable, and that stroke should now be regarded as a medical emergency.

STROKE COMES ON RAPIDLY

The WHO has defined stroke as a clinical syndrome characterised by rapidly developing symptoms and/or signs of focal, and at times global (for patients in coma), loss of cerebral function, with symptoms lasting more than 24 hours or leading to death, with no apparent cause other than that of vascular origin.

\section{STROKE HAS A POOR PROGNOSIS}

The outcome after stroke is crucially dependent on the extent and site of the brain damage, as well as the patient's age and prestroke health status. ${ }^{8}$ The case fatality rates after a first ever stroke (all types combined) are $12 \%$ at 7 days, $19 \%$ at 30 days, and $31 \%$ at 1 year'; haemorrhagic stroke carries a higher risk of death than ischaemic stroke. ${ }^{9}$ Deaths occurring within the first week after stroke are mostly due to the direct effects of cerebral damage ${ }^{81011}$; later on, the complications of immobility (for example, bronchopneumonia, venous thromboembolism) and cardiac events become increasingly common. ${ }^{89}$ About $20 \%$ of those with first ever stroke will be dependent on another person for everyday activities (for example, washing, dressing, mobility) at 12 months, and $50 \%$ will be independent. ${ }^{12}$ The risk of a recurrent stroke in survivors is about $10 \%$ to $16 \%$ within the first year, thereafter falling to about $5 \%$ per year. ${ }^{13}$ The relative risk of death in stroke survivors is about twice the risk of people in the general population, ${ }^{9}$ and this risk persists for several years; many of these deaths are due to other vascular problems (for example, ischaemic heart disease or peripheral arterial disease). This emphasises the importance of targeting these areas as well as stroke when we consider secondary prevention strategies.

STROKE PATIENTS MAY REQUIRE IMMEDIATE TREATMENT

There are several reasons why many patients require urgent inpatient care after an acute stroke. Firstly, stroke may lead to various potentially life threatening complications such as airway obstruction and respiratory failure, swallowing problems with the risk of aspiration, dehydration and malnutrition, venous thromboembolic complications, seizures, and infections. ${ }^{14-17}$ These may arise within hours of stroke onset and require early assessment and intervention so that they can be anticipated, prevented, and treated. Furthermore, although stroke has represented an area of therapeutic nihilism for many years, various acute and potentially effective treatments (medical and surgical) are now becoming available.

\section{Assessment}

Early assessment allows the formulation of an accurate and early diagnosis (as stroke is primarily a clinical diagnosis, the sooner a physician can elicit a history, the more likely it is to be reliable), the organisation of relevant and cost effective investigations, and the initiation of appropriate secondary prevention (which is likely to be most effective early on, when the risk of recurrence is highest). However, as with any medical emergency, the first priority in assessing a patient after a suspected stroke is to identify and treat any immediately life threatening complications. For stroke, this will usually be an obstructed airway, respiratory failure in a comatose patient, or an acute circulatory disturbance. Once the patient is stable, we apply a systematic, staged approach to making the diagnosis and formulating a management plan. This initial assessment should consider the following questions:

\section{(1) IS THIS A VASCULAR EVENT?}

The diagnosis depends crucially on an accurate history, taken from the patient or carer. We ask ourselves the following questions to help decide whether it was a vascular event.

- Are the neurological symptoms focal rather than non-focal?

- Are the focal neurological symptoms negative (loss of function) rather than positive (for example pins and needles rather than numbness)?

- Was the onset of the focal symptoms sudden?

- Were the focal symptoms maximal at onset (coming on over minutes to hours) rather than progressive (evolving over hours to days)?

If the answer to all these questions is yes, then a vascular cause (either cerebral ischaemia or haemorrhage) is very likely. Of course presentations vary. Occasional patients have symptoms or signs which are not easily localised (for example, memory impairment, confusion, or a reduced conscious level), symptoms may be positive (for example, movement disorders), and many patients describe symptoms evolving over hours or even days. These exceptions simply make the clinical diagnosis less certain and should lead to early investigation to exclude alternative diagnoses which require different urgent treatment (for example, hypoglycaemia, non-convulsive seizures, cerebral infection, or subdural haematoma).

In addition, we consider the context in which the event has occurred. Strokes are uncommon in the young, and as about $80 \%$ of stroke patients have at least one vascular risk factor at presentation, ${ }^{18}$ the absence of risk factors should lead one to be slightly more sceptical about a diagnosis of stroke. Accurate diagnosis in the hyperacute phase (less than 6 hours from onset) is often difficult because symptoms and signs may be changing rapidly. The introduction of acute therapies which need to be 
administered within this time window suggests that early and accurate diagnoses will become increasingly important.

(2) WHICH PART OF THE BRAIN IS AFFECTED? In reaching a diagnosis of stroke one inevitably makes some assessment of where in the brain the lesion might be. However, it may be useful to further subclassify the stroke as this may give clues to the likely underlying cause, allow more cost effective investigations, and help in predicting both the risk of recurrence and functional outcome. Although there are many subclassification systems available, we use the system developed from the Oxfordshire Community Stroke Project (table 1), ${ }^{19}$ which works well at the bedside. ${ }^{20-25}$

\section{(3) IS IT A HAEMORRHAGIC OR ISCHAEMIC}

STROKE?

Distinguishing between a haemorrhagic and ischaemic stroke is important in terms of acute management, prognosis, and secondary prevention. In white people, about $80 \%$ of first ever strokes are ischaemic. ${ }^{326}$ Although various scoring systems have been devised to help differentiate between infarction and haemorrhage, none provide sufficient accuracy to guide treatment. ${ }^{27}$ The only reliable method of differentiating is early brain imaging. In many countries, this is best performed by CT. Lumbar puncture may be useful in confirming subarachnoid haemorrhage if the brain imaging is equivocal, but it has no place in differentiating ischaemic and haemorrhagic stroke.

\section{Computed tomography}

Intracerebral blood immediately appears as an area of high density on CT, but thereafter decreases so that haemorrhagic lesions will eventually appear either isodense or hypodense, and thus be indistinguishable from an infarct. ${ }^{28}$ Smaller haemorrhages may become isodense within days although usually this process takes weeks. Computed tomography in the hyperacute stage of an ischaemic stroke is often normal although there may be subtle changes which are easily overlooked by the inexperienced observer. Infarcts are most easily seen on CT after a few days or in the chronic phase, when they may become markedly hypodense and well defined, although up to $50 \%$ of patients with a clinically definite stroke never have an appropriate lesion identified on CT. ${ }^{29}$ Although early CT will reliably identify intracerebral haemorrhage, the distinction between a primary intracerebral haemorrhage $(\mathrm{PICH})$ and haemorrhagic transformation of an infarct (HTI) is unreliable and difficult. The frequency and clinical relevance of HTI is uncertain, and radiologically ranges from small petechial haemorrhages to frank haematoma, which may or may not be accompanied by clinical deterioration. An HTI can occur very early, ${ }^{30}$ and the only definitive way of diagnosing HTI is to have an earlier scan excluding haemorrhage, so we recommend scanning as early as possible, ideally at the time of initial assessment.

\section{Magnetic resonance imaging}

Magnetic resonance imaging is probably more sensitive than CT for detecting stroke, particularly lacunar strokes and those occurring in the posterior fossa. However, even MRI can be normal in clinically definite stroke. ${ }^{31}$ Certain MRI techniques, such as diffusion weighted imaging, are very sensitive at highlighting the "culprit" lesion, which may be useful when several areas of abnormality are shown. The differentiation between an ischaemic and haemorrhagic stroke on MRI in the first few days is less easy for the non-expert than with CT but MRI can help diagnose intracerebral haemorrhage months or even years after the event when CT shows only a hypodense area indistiguishable from an infarct. However, physicians in many countries do not have urgent access to MRI, and it is currently a difficult technique to use safely and satisfactorily in many acutely ill patients; consequently, CT is likely to remain the principal imaging technique for stroke patients for the foreseeable future. Where available, MRI, including the use of specific sequences such as diffusion weighted imaging and MR angiography, may add significantly to the understanding of stroke mechanisms.

(4) WHAT CAUSED THIS STROKE?

The list of potential causes is long, and obviously differs for ischaemic ${ }^{32}$ and haemor-

Table 1 The Oxfordshire Community stroke subclassification system ${ }^{19}$

Total anterior circulation syndrome (TACS); implies a large cortical stroke in middle cerebral, or middle and anterior cerebral artery territories

A combination of:

- New higher cerebral dysfunction (eg dysphasia, dyscalculia, visuospatial disorder) AND

- Homonymous visual field defect AND

- An ipsilateral motor and/or sensory deficit involving at least two out of three areas of the face, arm or leg

Partial anterior circulation syndrome (PACS); implies cortical stroke in middle or anterior cerebral artery territory

Patients with two out of the three components of the TACS OR new higher cerebral dysfuction alone OR a motor/sensory deficit

more restricted than those classified as a LACS (eg isolated hand involvement).

Lacunar syndrome (LACS); implies a subcortical stroke due to small vessel disease

- Pure motor stroke

- Pure sensory stroke

- Sensorimotor stroke

- Ataxic hemiparesis

nb: evidence of higher cortical involvement or disturbance of consciousness excludes a lacunar syndrome

Posterior circulation syndrome (POCS)

- Ipsilateral cranial nerve palsy with contralateral motor and/or sensory deficit

- Bilateral motor and/or sensory deficit

- Disorder of conjugate eye movement

- Cerebellar dysfuction without ipsilateral log-tract involvement

- Isolated homonymous visual field defect 
rhagic stroke. ${ }^{33}$ In individual patients, even after extensive investigation it may be difficult to establish the cause: many will have competing causes (for example, $\mathrm{AF}$ and carotid disease). Thus in practice, the precise cause of stroke is often uncertain. Accepting this, we estimate that about $50 \%$ of ischaemic strokes are due to atherothromboembolism, $25 \%$ due to intracranial small vessel disease, and $20 \%$ due to cardiac embolism, with only $5 \%$ due to rarer causes. Most haemorrhagic strokes are thought to be due to small vessel disease (often associated with hypertension), although amyloid angiopathy commonly underlies lobar haemorrhages; vascular abnormalities such as aneurysms and arteriovenous malformations may also underlie haemorrhage, and the risk of haemorrhage with anticoagulant drugs increases with the international normalised ratio. The history and examination may provide important aetiological clues (for example, the use of oral anticoagulants, the presence of an irregular pulse, or heart murmur). Unusual causes are considerably more likely in younger patients (for example, evidence of drug misuse, or recent cervical trauma precipitating arterial dissection). Our approach to investigation aims to be reasonably cost effective. We perform some simple investigations (full blood count, erythrocyte sedimentation rate, plasma glucose, urea and electrolytes, random plasma cholesterol, urinalysis, 12-lead ECG, and brain CT) in all patients in whom we are considering active management, even if our clinical assess- ment strongly suggests a common cause. These tests may identify important modifiable risk factors as well as highlighting the possibility of a rarer, unexpected cause of stroke (for example, infective endocarditis, giant cell arteritis, or thrombocythaemia) which may coexist with either cardiac or degenerative vascular disease. We reserve other more specialised tests ${ }^{32}$ for patients in whom the cause of stroke is not clear (for example, young patients (less than 50 years), or those without risk factors), for those with clinical features of a rare cause, or where simple investigations show an abnormality (table 2).

\section{(5) WHAT ARE THIS PARTICULAR PATIENT'S} PROBLEMS?

A full assessment by the various members of the multidisciplinary team should be able to identify existing problems and anticipate future ones so that a problem and goal orientated management plan can be constructed. As well as assessing individual impairments and disabilities which may lead to specific interventions (for example, positioning and physiotherapy for hemiparesis), it is important not to ignore but to treat the less specific but unpleasant symptoms such as headache, vomiting, hiccups, vertigo, constipation, and the aches and pains which so often accompany prolonged immobility. Here, we will briefly discuss some of the most common early problems which account for significant mortality and morbidity, and which are most relevant to the physician. The list is not exhaustive and does

Table 2 Second line investigations in selected stroke patients

\begin{tabular}{|c|c|c|}
\hline Investigation & Indications & Disorders suggested \\
\hline Liver function tests & Fever, malaise, raised erythrocyte, ESR, malignancy & $\begin{array}{l}\text { Giant cell arteritis, infective and non-bacterial } \\
\text { thrombotic endocarditis }\end{array}$ \\
\hline Calcium & $\begin{array}{l}\text { Hypercalcaemia may rarely cause recurrent focal } \\
\text { symptoms }\end{array}$ & Hypercalcaemia \\
\hline $\begin{array}{l}\text { Activated partial thromboplastin time, dilute Russell's } \\
\text { viper venom time, anticardiolipin antibody, } \\
\text { antinuclear and other antibodies }\end{array}$ & $\begin{array}{l}\text { Young patient, previous or family history of venous } \\
\text { thrombosis, recurrent miscarriages, } \\
\text { thrombocytopenia, cardiac valve vegetations, livedo } \\
\text { reticularis, raised ESR, malaise, positive VDRL }\end{array}$ & $\begin{array}{l}\text { Antiphospholipid antibody syndrome, systemic } \\
\text { vasculitis, systemic lupus erythematosus }\end{array}$ \\
\hline $\begin{array}{l}\text { Protein } \mathrm{C} \text { and } \mathrm{S} \text {, antithrombin } \mathrm{III} \text {, activated protein } \mathrm{C} \\
\text { resistance, thrombin time }\end{array}$ & $\begin{array}{l}\text { Previous or family history of thrombosis (usually } \\
\text { venous) of young onset }\end{array}$ & Deficiency states \\
\hline Serum proteins and electrophoresis, plasma viscosity & Raised ESR & $\begin{array}{l}\text { Paraproteinaemias, nephrotic syndrome, cardiac } \\
\text { myxoma }\end{array}$ \\
\hline Haemoglobin electrophoresis & AfroCaribbean patients & $\begin{array}{l}\text { Sickle cell trait or disease, other } \\
\text { haemoglobinopathies }\end{array}$ \\
\hline Blood cultures & $\begin{array}{l}\text { Fever, cardiac murmur, haematuria, deranged LFTs, } \\
\text { raised ESR, malaise }\end{array}$ & Infective endocarditis \\
\hline VDRL, HIV serology & Young, unexplained or "at risk" & Neurosyphilis, AIDS \\
\hline Serum homocysteine, urinary amino acids & $\begin{array}{l}\text { Marfanoid habitus, high myopia, dislocated lenses, } \\
\text { osteoporosis, mental retardation, young }\end{array}$ & Homocystinuria \\
\hline Leucocyte $\alpha$-galactosidase A & $\begin{array}{l}\text { Corneal opacities, cutaneous angiokeratomas, } \\
\text { paraesthesias and pain, renal failure }\end{array}$ & Fabry's disease \\
\hline Blood/CSF lactate, mitochondrial DNA analysis & $\begin{array}{l}\text { Young, basal ganglia calcification, epilepsy, } \\
\text { parieto-occipital ischaemia, migraine }\end{array}$ & MELAS/mitochondrial cytopathy \\
\hline Drug screen (blood or urine) & "At risk" patient, no other cause & Drug induced stroke (amphetamine, cocaine, etc) \\
\hline Chest radiography & $\begin{array}{l}\text { Hypertension, finger clubbing, cardiac murmur or } \\
\text { abnormal ECG, young }\end{array}$ & Calcified valves, enlarged heart, pulmonary AVM \\
\hline Carotid ultrasound/MR angiography & $\begin{array}{l}\text { Carotid distribution stroke in patient suitable for } \\
\text { surgery }\end{array}$ & Cervical internal carotid stenosis \\
\hline $\begin{array}{l}\text { Cerebral angiography (intra-arterial digital subtraction } \\
\text { or MR) }\end{array}$ & $\begin{array}{l}\text { Young unexplained stroke, especially associated with } \\
\text { pain or trauma, suspected arteritis, AVM or } \\
\text { aneurysm }\end{array}$ & Arterial dissection, vascular abnormality \\
\hline Transthoracic echocardiography & Suspected cardioembolism & Cardioembolism \\
\hline Transoesophageal echocardiography & $\begin{array}{l}\text { Suspected cardioembolism when TTE negative } \\
\text { (eg endocarditis, atrial septal aneurysm), aortic } \\
\text { dissection or atheroma, patent foramen ovale }\end{array}$ & $\begin{array}{l}\text { Cardioembolism, aortic dissection or atheroma, } \\
\text { paradoxical embolism }\end{array}$ \\
\hline 24 hour ECG & $\begin{array}{l}\text { Palpitations, suspicious resting ECG, clinical } \\
\text { suspicion }\end{array}$ & Intermittent $\mathrm{AF}$, heart block \\
\hline Temporal artery biopsy & $\begin{array}{l}\text { Older }(>60) \text {, jaw claudication, headache, polymyalgia, } \\
\text { malaise, anaemia, raised ESR }\end{array}$ & Giant cell arteritis \\
\hline
\end{tabular}

ESR=erythrocyte sedimentation rate; VDRL=venereal disease research laboratory test; AVM=arteriovenous malfunction; LFT=liver function test; $\mathrm{TTE}=$ transthoracic echocardiography; $\mathrm{AF}=$ atrial fibrillation. 
not include important problems which may arise later on (for example, painful shoulders or depression). ${ }^{34}$

\section{Airway and breathing}

Although stroke may cause various abnormal breathing patterns ${ }^{35}{ }^{36}$ (for example, periodic respiration or hyperventilation), an intermittently obstructed airway in patients with a decreased level of consciousness may mimic this and should be excluded. The presence of significant hypoxia should stimulate a search for possible causes (for example, pulmonary oedema, pulmonary embolism, or infection). It seems reasonable to attempt to correct this with supplemental oxygen, but we do not advocate routine supplemental oxygen for all patients. We increasingly use pulse oximetry in the acute phase to alert us to significant oxygen desaturation.

\section{Circulation}

Hypotension is relatively uncommon in stroke patients; if it does occur, it is usually secondary to coexistent heart disease (arrhythmias, heart failure or acute myocardial infarction), dehydration, or sepsis. As cerebral autoregulation is disturbed after stroke, with the result that cerebral blood flow becomes directly dependent on systemic blood pressure, urgent correction is required. By contrast, hypertension is extremely common after stroke, even in patients without pre-existing hypertension. ${ }^{37} 38$ Although some authorities recommend early pharmacological lowering of raised blood pressure, given the current absence of any convincing evidence of the effectiveness of such a policy, ${ }^{39-42}$ and the recognised potential dangers of hypotension, ${ }^{43}$ we only give hypotensive drugs early (within the first 72 hours) to patients with features of accelerated hypertension or hypertensive encephalopathy, or acute aortic dissection. We usually continue any previous antihypertensive medication a patient may have been taking, provided they are not hypotensive and can safely swallow the tablets. There is uncertainty about when hypotensive drugs should be started after the acute phase; in many cases a raised blood pressure falls spontaneously in the days after an acute stroke. We usually delay consideration of long term drug therapy for at least a week, although we acknowledge that some physicians would start therapy earlier.

\section{Raised intracranial pressure}

Although intracranial pressure may rise very rapidly after haemorrhagic stroke (due to the space occupying effects), it usually takes at least 48 hours, and often longer, to manifest after an ischaemic stroke (except in the unusual case of a cerebellar or brainstem infarct obliterating the CSF pathways and resulting in hydrocephalus). Although treatments such as mannitol, ${ }^{44}$ hyperventilation, and even decompressive craniectomy ${ }^{45}$ undoubtedly reduce intracranial pressure, it is unclear whether such aggressive interventions are associated with improved survival with acceptable quality of life. In selected patients who are deteriorating rapidly and who are judged to have some chance of a reasonable recovery we do consider transfer to an intensive care unit for aggressive management of raised intracranial pressure. However it is important to remember that early deteriorating conscious level is usually a very poor prognostic indicator, and such intensive treatment in an elderly patient with significant other neurological deficits is, in our view, rarely justified.

\section{Stroke in evolution}

After the onset of symptoms, some patients continue to deteriorate over several hours or days. This is variably referred to as progressing or evolving stroke. ${ }^{46}{ }^{47}$ Such patients require prompt reassessment and investigation, as there is a wide range of potential causes of deterioration, some of which may be reversible (table 3). If we suspect that the cause is progressive thromboembolism, we might use intravenous heparin despite the lack of evidence supporting its effectiveness (see below).

\section{Swallowing, hydration, and nutrition}

Dysphagia $^{48}$ and poor nutrition ${ }^{49-52}$ are common after stroke and may lead to further complications. ${ }^{53}$ All patients should have a bedside swallowing assessment ${ }^{545}$ as part of their initial assessment by a suitably trained member of the multidisciplinary team. The gag reflex is an unreliable indicator of swallowing ability and should not be used for this purpose. ${ }^{54}$ The bedside assessment should lead to a decision regarding whether the patient is safe to swallow or not, and should be written down and clearly communicated to the nursing staff. For patients with an unsafe swallow, fluids should be prescribed (either intravenous or nasogastric), and arrangements made for further assessment by a speech and language therapist. The role of early enteral tube feeding, its timing, and whether this is best delivered via a nasogastric tube or percutaneous endoscopic gastrostomy remains unclear, and is the subject of an ongoing multicentre trial. ${ }^{56}$ Percutaneous endoscopic gastrostomy tube feeding is clearly the best option where prolonged tube feeding is necessary. However in the early stages, where the advantages and disadvantages of early versus delayed tube feeding, and the optimal type of tube are unclear, we randomise our patients in this trial.

\section{Table 3 Causes of deterioration after stroke}

Neurological:

- Progression/completion of stroke

- Extension/early recurrence

- Haemorrhagic transformation of an infarct

- Developing cerebral oedema*

- Obstructive hydrocephalus ${ }^{\star}$

- Epileptic seizures ${ }^{\star}$

- Incorrect diagnosis ${ }^{\star}$

Non-neurological:

- Infection ${ }^{\star}$

- Metabolic derangement ${ }^{\star}$

- Drugs`

- Hypoxia

- Hypercapnoea ${ }^{\star}$

$\star$ Potentially reversible causes. 
Glycaemic control

Hypoglycaemia, although unusual after a stroke, should always be excluded on admission, as it may mimic stroke perfectly, and delay in its correction can lead to permanent disability or even death. Hyperglycaemia is much more common and has been attributed to previously recognised or occult diabetes, or part of an acute stress response. ${ }^{57}$ Hyperglycaemia is associated with poor outcomes and although work in animal models suggests that this association may be causal, it may simply reflect the severity of the stroke or underlying vascular disease. Thus it is unclear how aggressively hyperglycaemia should be corrected and at least one randomised controlled trial is now in progress. ${ }^{58}$ Until further evidence is available our policy is to use a glucose, potassium, and insulin infusion to correct blood sugars persistently above $15 \mathrm{mmol} / \mathrm{l}$, and to treat even at lower levels provided there are adequate facilities for close monitoring to minimise the risk of hypoglycaemia.

Pyrexia

This may be due to infection preceding the stroke (consider endocarditis and encephalitis), the stroke itself, or most commonly a complication such as a chest or urinary infection, or venous thromboembolism. Obviously the underlying cause should be sought and treated but it is probably sensible to try to reduce the temperature using simple means (for example, antipyretic drugs) in any case as this is likely to make the patient more comfortable and there is a possibility, based on animal models and the observation that raised temperatures are associated with poor outcomes in patients, ${ }^{59-62}$ that a raised temperature may exacerbate any ischaemic cerebral damage. ${ }^{63}$ There are no published randomised trials of cooling therapy yet, for patients with either a raised or normal temperature, but small open studies have started to explore the use of cooling. ${ }^{64}$

\section{Pressure areas}

Decubitus ulcers or pressure sores are an entirely avoidable complication, assuming that they did not develop before medical help was sought. When they do occur, they are painful, slow the patient's recovery, and may sometimes be fatal. Prevention relies on an early assessment of the patient's risk, expert nursing care, and the judicious use of specialised cushions and mattresses. ${ }^{65}$

\section{Bladder management}

Incontinence of urine is common in the first few days and a source of major distress for patients and their carers. ${ }^{66}$ Usually it can be attributed to several factors including impaired sphincter control, immobility, communication problems, constipation, pre-existing prostatic or gynaecological problems, inadequate nursing, infection, confusion, and impaired consciousness. Obviously the cause or causes should be identified and rectified if possible. Most patients can be managed using absorbent pads, external urinary devices, and regular toi- leting regimes. If these are impractical, and transfers very difficult and the patient's pressure areas are causing concern, we insert an indwelling catheter despite the risk of infection and trauma. Incontinence often resolves spontaneously within the first week or two, so it is wise to try removing the catheter if it seems likely that things will have improved. For patients with persisting incontinence further investigation with bladder ultrasound or postmicturition catheterisation may be useful to assess bladder contractility and outflow. Urinary retention, particularly in men, is common and easily missed in patients with communication problems.

\section{Venous thromboembolism prophylaxis}

Studies using radiolabelled fibrinogen leg scanning suggest that deep venous thrombosis (DVT) occurs in over $50 \%$ of patients with hemiplegia. ${ }^{67}$ However, clinically apparent DVT probably occurs in fewer than $5 \% .{ }^{14}$ Similarly, although postmortem series have identified pulmonary embolism in a large proportion, ${ }^{68}$ clinically evident pulmonary embolism occurs in less than $2 \%,{ }^{15} 173469$ although some pulmonary embolism may be unrecognised. The impact of venous thromboembolism after stroke is therefore unclear.

There are two strategies for prevention of venous thromboembolism; physical interventions (for example, early mobilisation and compression stockings), and antithrombotic drug therapy. The evidence to support the use of compression stockings comes from randomised control trials in the perioperative period $^{70}$ which may not be generalisable to stroke because in stroke the stockings are applied after the onset of paralysis, and immobilisation is often prolonged. Also compression stockings, apart from being uncomfortable and time consuming to apply, can occasionally cause gangrene in patients with poor peripheral circulation. It therefore seems reasonable to recommend early mobilisation wherever possible and compression stockings (usually full length) for patients at high risk of DVT (those who are immobilised, or who have a history of DVT). However given the difficulties and risk we think that further trials to evaluate their effectiveness in stroke patients are justified.

There is reasonable evidence that aspirin reduces the risk of DVT in several clinical situations ${ }^{71}$ and it also has a small but beneficial effect on the long term outcome of patients with ischaemic stroke (see later), so we use this routinely. Although low dose subcutaneous heparin significantly reduces the risk of DVT and pulmonary embolism, this effect is offset by the complications of haemorrhagic transformation and extracranial bleeding, such that at 6 months the average patient with ischaemic stroke has no greater chance of surviving free of dependency if treated with heparin. ${ }^{72}$ We occasionally use heparin (standard unfractionated, at a dose of 5000 units twice daily subcutaneously) in patients we judge to be at particularly high risk of venous thromboembolism (for example, those with a history of previous 
DVT/pulmonary embolism) and low risk of HTI (for example, lacunar infarction).

Epileptic seizures

Early seizures (within 2 weeks of stroke) occur in about $5 \%$ of patients. ${ }^{73-75}$ They are more common in haemorrhagic stroke and large infarcts involving the cerebral cortex. Seizures should prompt a review of the diagnosis of stroke (could the focal symptoms be secondary to postictal paralysis or encephalitis?), and a search for precipitating factors (for example, alcohol withdrawal, drugs, metabolic disturbance, or infection). After treating the seizures, we would then reappraise the severity of stroke, as this is notoriously difficult in the presence of seizures. We have occasionally misdiagnosed stroke in patients with non-convulsive seizures, which requires an EEG for definitive diagnosis. The treatment of poststroke seizures is no different from other forms of secondary epilepsy. ${ }^{76}$

\section{Specific treatments for acute ischaemic stroke}

In the United Kingdom few treatments aimed specifically at the ischaemic brain lesion are routinely used. However, many treatments are used routinely in other countries, and evidence is accruing that certain treatments may improve outcome in selected patients. We therefore consider some of these further and review the available evidence to support their use. Before doing so, it may be helpful to consider briefly the main pathophysiological features of an ischaemic stroke; for a more detailed review, we refer readers elsewhere. ${ }^{77} 78$

PATHOPHYSIOLOGY OF ISCHAEMIC STROKE

Ischaemic stroke usually occurs due to occlusion of a cerebral artery, or less often a reduction in perfusion distal to a severe stenosis. As cerebral blood flow falls, neuronal function is affected in two stages. Initially, as blood flow falls below a critical threshold of about $20 \mathrm{ml}$ blood/100 g brain/min (normal being over $50 \mathrm{ml} / 100 \mathrm{~g} / \mathrm{min}$ ), loss of neuronal electrical function occurs. Crucially, this is a potentially reversible stage. Irreversible damage occurs within minutes as blood flow falls below a second critical threshold of $10 \mathrm{ml} / 100 \mathrm{~g} / \mathrm{min}$; below this level, aerobic mitochondrial metabolism fails, and the inefficient anaerobic metabolism of glucose takes over, rapidly leading to lactic acidosis. Consequently, the normal energy dependent cellular ion homeostasis fails, resulting in potassium leaking out of the cell, and sodium and water entering the cell, leading to cytotoxic oedema. Calcium also enters the cell, exacerbating mitochondrial failure. This loss of cellular ion homeostasis leads to neuronal death.

The identification of these two stages of neuronal failure has led to the concept of the ischaemic penumbra - that is, an area of brain which has reached the reversible stage of electrical failure, but has not yet passed onto the second irreversible stage of cellular homeostatic failure. In theory therefore, this tissue could be "rescued", either by early reperfusion (using agents to dissolve the acute thrombotic lesion and restore normal blood flow), or by administering agents which could protect these potentially viable neurons from further damage (neuroprotection); the combination of reperfusion and neuroprotection would seem a logical conclusion. ${ }^{79}$ Although there is evidence that the concept of the ischaemic penumbra is valid, ${ }^{80}$ it remains unclear how long ischaemic human brain might survive-in other words, the time window for intervention is unknown. It seems likely that the duration of any time window will vary between individual patients, and it will be increasingly important to identify the factors which influence it.

Should the mechanism of the cerebral ischaemia influence management? Some experts think that certain specific causes of ischaemia, such as basilar artery thrombosis or arterial dissection, warrant specific interventions, most commonly anticoagulation. There is no convincing evidence to support these views, and therefore we tend to treat them the same as we would any other form of ischaemic stroke. A recent randomised trial of the use of anticoagulants in cerebral venous thrombosis indicated a non-significant favourable effect. ${ }^{81}$

\section{THROMBOLYSIS}

Despite having been used sporadically for over 40 years, evidence for the effectiveness of thrombolytic therapy in acute ischaemic stroke has only recently become available. A systematic review of the results of 12 of the 14 completed randomised controlled trials in the post -CT era suggests that although thrombolytic therapy (with recombinant tissue plasminogen activator, streptokinase, or urokinase) is associated with about 70 symptomatic (about 50 fatal) intracranial bleeds per 1000 patients treated, its use is associated with perhaps 65 more patients surviving free of dependency at 3 to 6 months poststroke (fig 1). ${ }^{82}$ Even more compelling are the updated analyses of treatment within the first 3 hours. ${ }^{83}$ These demonstrate less risk of early intracranial haemorrhage and early death, and greater long term net benefit (130 extra patients alive and independent per 1000 treated). How practicable the widespread use of thrombolysis will be (particularly for a condition which has not traditionally been thought of as an emergency) remains uncertain, although some units have published impressive figures. ${ }^{84}$ Recombinant tissue plasminogen activator ( $r$-TPA) is now licensed in the United States, and a European licence is likely to be granted in the near future; therefore, it seems reasonable to consider using r-TPA in patients presenting within 3 hours, and who are similar to the patients included in the trials, provided there is a stroke service which can ensure its safe administration (table 4). Our view is that further trials are required to establish the balance of risks and benefits in a broader range of patients presenting at different stages, with differing severities and types of ischaemic stroke, different risk factors, and differing scan appearances. Many of the eligibility criteria currently in place are arbitrary and are not based on any reliable evidence. If a larger proportion of patients were eligible for treat- 
Table 4 Suggested guidelines for the use of intravenous r-TPA in ischaemic stroke [99]

- Intravenous r-TPA should be considered in all patients with a proved ischaemic stroke presenting within 3 hours of onset

- Recommended dose is $0.9 \mathrm{mg} / \mathrm{kg}$, up to a maximum of $90 \mathrm{mg}$, the first $10 \%$ as a bolus, the rest as an infusion over 60

minutes

- Thrombolysis should be avoided in cases where the CT suggests early changes of major infarction (for example, sulcal

effacement, mass effect, or oedema)

- Thrombolytic therapy should only be administered by physicians with expertise in stroke medicine, who have access to a suitable stroke service, with facilities for identifying and managing haemorrhagic complications

- Exclusion criteria: use of oral anticoagulants, or INR greater than 1.7; use of heparin in preceding 48 hours or prolonged partial thromboplastin time; platelet count less than $100000 \mathrm{~mm}^{3}$; stroke, or serious head injury in the previous 3 months; major surgery within previous 14 days; pretreatment systolic blood pressure greater than $185 \mathrm{~mm} \mathrm{Hg}$ or diastolic greater than $110 \mathrm{~mm} \mathrm{Hg}$; rapidly improving neurological condition; mild isolated neurological deficits; previous intracranial haemorrhage; blood glucose greater than $22 \mathrm{mmol} / 1(400 \mathrm{mg} / \mathrm{dl})$ or less than $2.8 \mathrm{mmol} / 1(50 \mathrm{mg} / \mathrm{dl})$; seizure at stroke onset; gastrointestinal or urinary bleeding within previous 21 days; or recent myocardial infarction

- Caution is advised before giving r-TPA to patients with severe stroke (NIH stroke scale score> 22)

- Recommended that treatment and adverse effects discussed with patient and family before treatment.

ment the potential impact on the burden of stroke would be greater and it may then be easier to justify the major changes in the delivery of acute stroke services which are required.

ANTICOAGULANTS (INCLUDING STANDARD UNFRACTIONATED HEPARIN, LOW MOLECULAR WEIGHT HEPARINS, AND HEPARINOIDS)

A recent systematic review comparing immediate anticoagulant therapy with control in acute ischaemic stroke, including over 20000 patients, concluded that although anticoagulation started in the first day or two may reduce the risk of DVT and pulmonary embolism (see above) there were no short or long term benefits in terms of survival free of dependency $^{72}$ (fig 2). In addition, there was no evidence to support the use of anticoagulants in any specific patient category (for example, presumed cardioembolic stroke or vertebrobasilar stroke). The only situation in which we consider starting anticoagulation (with intra- venous, standard unfractionated heparin) is for patients with an evolving, ischaemic stroke established by CT which we consider is likely to be due to progressive thromboembolism, although there is no convincing evidence to justify this policy. The individual threshold for using early anticoagulation is very variable, and some physicians use anticoagulants for specific situations such as basilar artery thrombosis or intracardiac thrombus.

Although we know that oral anticoagulation with warfarin is effective in the secondary prevention of stroke in patients with atrial fibrillation, ${ }^{85} 86$ we have considerable difficulty deciding when to start warfarin after the primary event. Although not evidence based, we tend to delay longer (perhaps by 2 weeks) in patients with large ischaemic cerebral lesions, thinking that they are more likely to have ill effects (mainly haemorrhagic transformation) from anticoagulation. The question of whether to anticoagulate patients with other potential

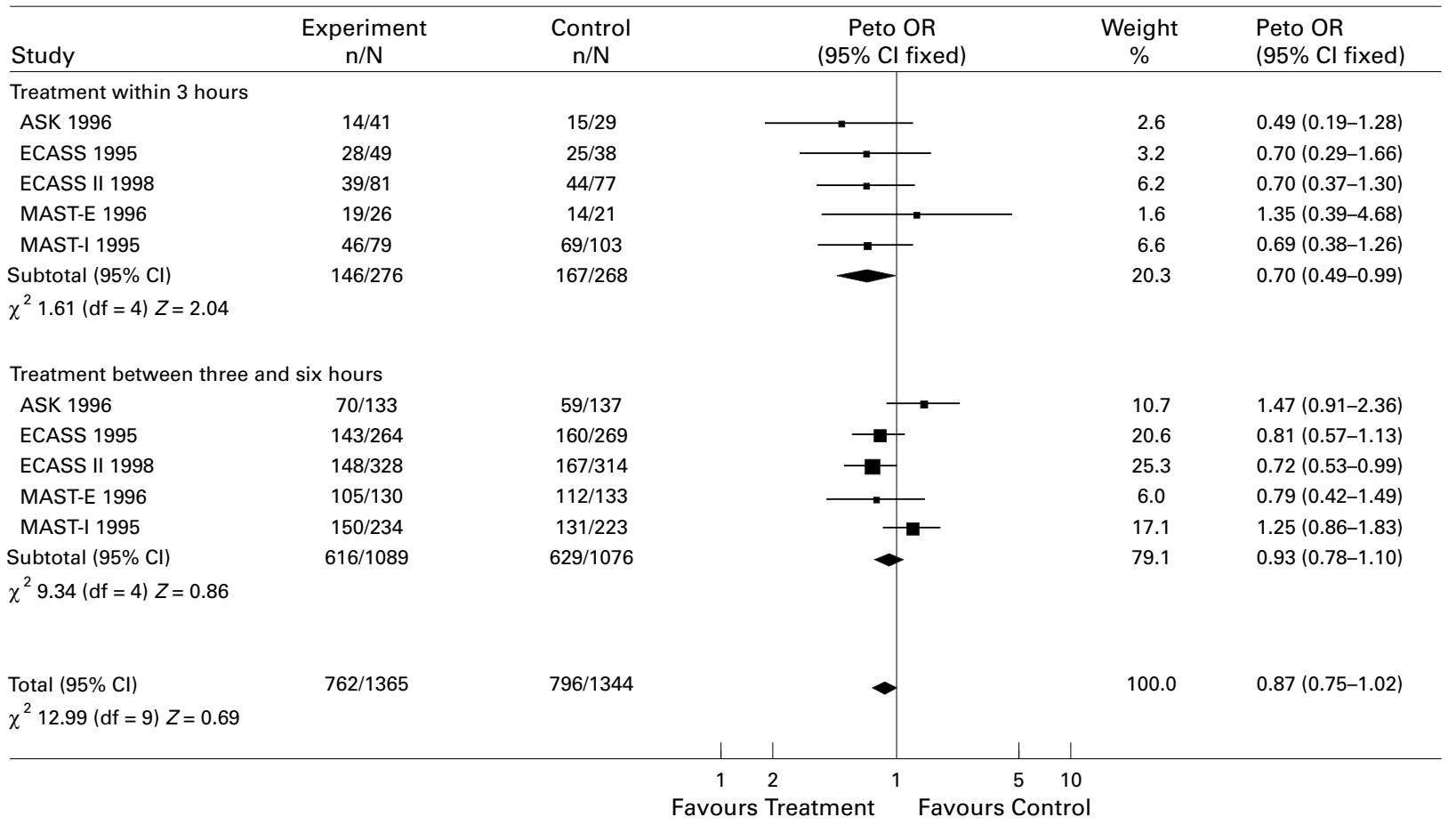

Figure 1 Results of a systematic review of the randomised trials of thrombolysis administered within 6 hours of the onset of ischaemic stroke established by CT. The estimate of treatment is expressed as an odds ratio (square, the size of the square indicating the statistical power of the estimate), and its $95 \%$ CI (horizontal bar); the diamond shapes provide estimates of the pooled trial results. OR=1 indicates a zero treatment effect, OR $<1$ indicates treatment better than control, and an OR>1 indicates treatment worse than control. Treatment with thrombolysis within 3 hours reduced death and dependency at the end of follow up. 


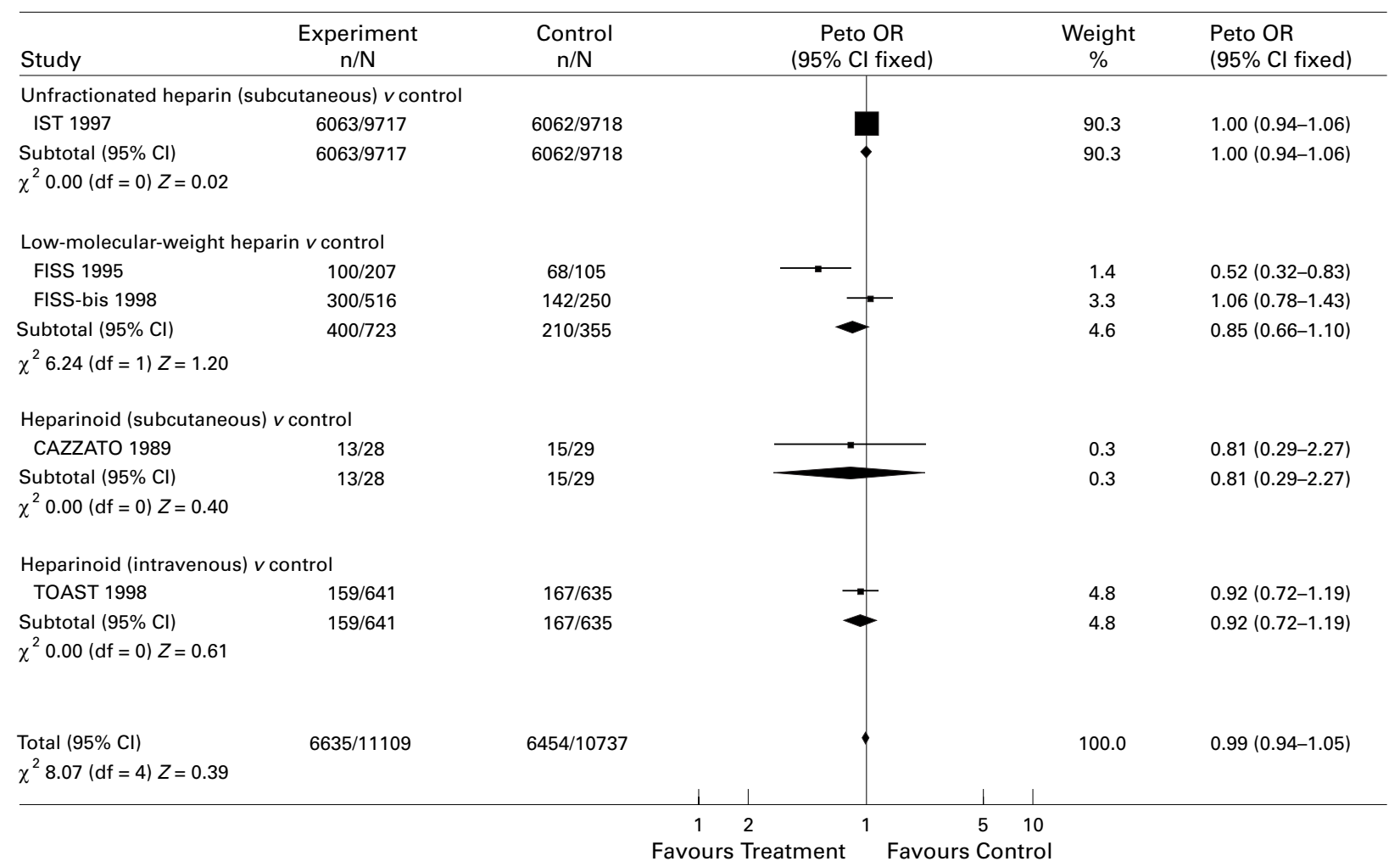

Figure 2 Results of a systematic review of the randomised trials of anticoagulants in acute presumed ischaemic stroke. The estimate of treatment is expressed as an OR (square, the size of the square indicating the statistical power of the estimate), and its 95\% CI (horizontal bar); the diamond shapes provide estimates of the pooled trial results. OR=1 indicates a zero treatment effect, $O R<1$ indicates treatment better than control, and OR $>1$ indicates treatment worse than control. There was no significant effect of anticoagulant treatment on death or dependency at the end of follow up ( $>1$ month).

cardioembolic sources, such as mitral valve disease without $\mathrm{AF}$, is very difficult, with little evidence to guide the physician.

ASPIRIN

The pooled results of two very large randomised controlled trials comparing aspirin with placebo, concluded that medium dose aspirin (160-300 mg) started in the acute phase of an ischaemic stroke produces a small (13 fewer patients per 1000 dead or disabled) net benefit. ${ }^{87}$ Whether this benefit arose from an effect on the stroke itself or simply through earlier initiation of secondary prevention of stroke and other thrombotic complications is uncertain. We therefore start all patients on $300 \mathrm{mg}$ aspirin as soon as a CT has confirmed an ischaemic stroke unless there is a specific contraindication; we later discharge patients on a maintenance dose of 75 to $150 \mathrm{mg}$ per day.

NEUROPROTECTIVE AGENTS

To date, no neuroprotective agent has been conclusively shown to be effective, and a Cochrane review summarising the current data is due to be published ${ }^{88}$ Trials to evaluate the neuroprotective effects of magnesium (IMAGES), benzodiazepines (EGASIS), and other novel agents are in progress.

OTHER TREATMENTS

Numerous other treatments have been used for ischaemic stroke, and some have been subjected to randomised trials. However, there is currently no convincing evidence to support the routine use of any of them.
Treatment of haemorrhagic stroke

Various specific treatments designed to reduce intracranial pressure are often used for primary intracerebral haemorrhage, including osmotic agents such as mannitol, urea or glycerol, steroids, or hyperventilation; unfortunately there is no convincing evidence that these treatments improve outcome. In view of the lack of evidence we do not routinely use any specific medical therapy in haemorrhagic stroke, nor do we employ invasive devices, such as intraventricular catheters, to directly measure intracranial pressure. We would attempt to correct or reverse any clotting abnormality, including those patients on oral anticoagulant drugs, although this depends on the original indication for the anticoagulants (for example, prosthetic heart valves).

SURGERY FOR SUPRATENTORIAL PICH

A systematic review of open surgical drainage via a craniotomy concluded that this sort of surgery was positively harmful. ${ }^{89}$ However, safer surgical techniques are now available, in particular stereotactic aspiration, and the results of ongoing surgical trials are awaited. In a previously fit person with a large lobar intracerebral haemorrhage whose conscious level is falling we would refer to our neurosurgeons and encourage them to drain the haematoma. In this situation where the patients are expected to die unless action is taken the decisions are relatively easy. More difficult are those patients with lesions deep in the hemisphere and those with severe impairments but no reduction in conscious level. These patients we 
usually manage conservatively, or randomise into one of the ongoing trials of surgical treatment.

SURGERY FOR INFRATENTORIAL PICH

Although there is general agreement that surgical intervention in this situation may be life saving (so much so that a randomised controlled trial is unlikely to ever be done), there is considerable uncertainty about which patients might benefit the most, or even which procedure is optimal (haematoma evacuation versus ventricular decompression via a ventriculostomy, or both). We would always consider surgical intervention in any patient who was comatose, or whose conscious level was progressively deteriorating, and in whom other exacerbating causes had been excluded (table 3). Once brainstem reflexes have been absent for several hours however, death is inevitable. ${ }^{90}$

\section{Organisation of stroke services}

In the United Kingdom, between $40 \%$ to $70 \%$ of patients are admitted to hospital after a stroke $^{91-93}$ and mostly cared for by general internal physicians and geriatricians. In many other countries, admission rates exceed 90\% and larger proportions of patients are managed by neurologists. In the United States and many European countries, professional bodies recommend hospital admission for most if not all patients after an acute stroke. ${ }^{94} 95$ Many patients require hospital admission on nursing grounds alone, or because of diagnostic uncertainty. It is now accepted that most patients, whether admitted to hospital or not, need early access to hospital based facilities such as CT. ${ }^{96} 97$ Patients who have had a mild, nondisabling stroke may not require inpatient care, but do need rapid assessment (within a few days), the exceptions perhaps being very infirm or elderly patrients, and those already in institutionalised care. Widespread introduction of thrombolytic therapy for ischaemic stroke, with its narrow time window, will demand much improved organisation of prehospital care and the introduction of "fast tracking" within hospitals to avoid delays.

A systematic review of all randomised controlled trials evaluating stroke unit care demonstrated that patients managed in stroke units are significantly less likely to die, have severe disability, or require long term institutional care than those managed in general medical wards. ${ }^{98}$ Although this review is often quoted to justify the establishment of acute stroke units (those which admit patients directly for just a few days to facilitate acute treatment and intensive monitoring), it did not include such units. Thus the evidence from randomised controlled trials only applies to units which can also offer at least several weeks of care coordinated by a multidisciplinary team. Having said this we think that patients should have access to comprehensive and well organised services, whatever their need. An acute unit run by interested specialists is more likely to ensure high quality care and will facilitate the introduction of evidence based protocols for investigation and treatment, as well as further research. It seems likely that the sooner acute specific treatments can be given the more effective they will be ("time is brain"). It is likely that there will be increasing emphasis on systems of prehospital care which facilitate earlier transfer to an acute stroke unit. However, this must not inhibit the development of other aspects of the services (for example, rehabilitation) which have been shown to have important benefits for patients.

Therefore hospitals need to develop both inpatient and outpatient services in collaboration with primary care, which can respond rapidly. As an important adjunct to developing these services, the general public should be educated about the symptoms of stroke, and the importance of early presentation to medical services.

1 Harris AI. Handicapped and impaired in Great Britain. London: The Stationery Office, 1971.

2 Martin J, Meltzer H, Elliot D. Office of Populations, Martin J, Meltzer H, Elliot D. Office of Populations,
Censuses and Surveys surveys of disability in Great Britain report 1 . The prevalence of disability amongst adults. London: report 1 . The prevalence of disabil

3 Sudlow C, Warlow C. Comparing stroke incidence worldwide. What makes studies comparable? Stroke 1996;27: $550-8$.

4 The organisation of stroke services. In: Warlow CP, Dennis MS, Van Gijn J, et al, eds.Stroke. A practical guide to management. Oxford: Blackwell, 1996;598-631.

5 King's Fund Forum. Treatment of stroke. BMF 1988;297: $126-8$.

6 Secretary of State for Health. The Health of the Nation. A strategy for health in England. London: The Stationery Office, 1992;

7 Hatano S. Experience from a multicentre stroke register: a

preliminary report. Bull WHO 1976;54:541-53.
8 Bamford J, Dennis M, Sandercock P, et al. The frequency, causes and timing of death within 30 days of a first stroke: the Oxfordshire community stroke project. 7 Neurol Neurothe Oxfordshire community stroke Psychiatry 1990;53:824-9.
surg

9 Dennis MS, Burn JP, Sandercock PA, et al. Long-term surDennis MS, Burn JP, Sandercock PA, et al. Long-term sur-
vival after first-ever stroke: the Oxfordshire community vival after first-ever stroke: the Oxfordshi
stroke project. Stroke 1993;24:796-800.

10 Bounds JV, Wiebers DO, Whisnant JP, et al. Mechanisms and timing of deaths from cerebral infarction. Stroke 1981; 12:474-7

11 Silver FL, Norris JW, Lewis AJ, et al. Early mortality following stroke: a prospective review. Stroke 1984;15:492-6.

2 A practical approach to the management of stroke patients. In: Warlow CP, Dennis MS, Van Gijn J, et al, Stroke. A practical guide to management. Oxford: Blackwell, 1996;36084.

13 Burn J, Dennis M, Bamford J, et al. Long-term risk of recurrent stroke after a first-ever stroke. The Oxfordshire community stroke project. Stroke 1994;25:333-337.

14 Davenport RJ, Dennis MS, Wellwood I, et al. Complications after acute stroke. Stroke 1996;27:415-20.

15 Dromerick A, Reding M. Medical and neurological compliDromerick A, Reding M. Medical and neurological compli-
cations during inpatient stroke rehabilitation. Stroke 1994; 25:358-61.

16 Dobkin BH. Neuromedical complications in stroke patients transferred for rehabilitation before and after diagnostic related groups. Fournal of Neurological Rehabilitation 1987; $1: 3-7$.

17 Kalra L, Yu G, Wilson K, et al. Medical complications during stroke rehabilitation. Stroke 1995;26:990-4

8 Sandercock PA, Warlow CP, Jones LN, et al. Predisposing factors for cerebral infarction: the Oxfordshire community stroke project. $B M \mathcal{F} 1989 ; 298: 75-80$.

19 Bamford J, Sandercock P, Dennis M, et al. Classification and natural history of clinically identifiable subtypes of cerebral infarction. Lancet 1991;337:1521-6.

20 Anderson CS, Taylor BV, Hankey GJ, et al. Validation of a clinical classification for subtypes of acute cerebral

infarction. F Neurol Neurosurg Psychiatry 1994;57:1173-9. Lindgren A, Norvving B, Rudling $\mathrm{O}$, et al. Comparison of
clinical and neuroradiological findings in first ever stroke: a clinical and neuroradiological findings in first eve

22 Mead GE, Wardlaw JM, Dennis MS, et al. The validity of a Mead GE, Wardlaw JM, Dennis MS, et al. The validity of a
simple clinical classification for acute stroke [abstract]. Age Ageing 1998;27(suppl 1):69.

23 Al-Buhairi AR, Phillips SJ, Llewellyn G, et al. Prediction of infarct topography using the Oxfordshire community stroke project classification of stroke subtypes. F Stroke Cerebrovasc Diseases 1998;7:339-43.

24 Wardlaw JM, Dennis MS, Lindley RI, et al. The validity of a simple clinical classification of acute ischaemic stroke. $\mathcal{F}$ Neurol 1996;243:274-9.

25 Lindley RI, Warlow CP, Wardlaw JM, et al. Interobserver reliability of a clinical classification of acute cerebral infarction. Stroke 1993;24:1801-4.

26 Bamford J, Sandercock P, Dennis M, et al. A prospective study of acute cerebrovascular disease in the community: the Oxfordshire community stroke project $1981-6$. 2. Inci 
dence, case fatality rates and overall outcome at one year of cerebral infarction, primary intracerebral and subarachnoid haemorrhage. $\mathcal{F}$ Neurol Neurosurg Psychiatry 1990;53: $16-22$.

27 Weir CJ, Murray GD, Adams FG, et al. Poor accuracy of scoring systems for differential clinical diagnosis of intracranial haemorrhage and infarction. Lancet 1994;344:9991002 .

28 Dennis MS, Bamford JM, Molyneux AJ, et al. Rapid resolution of signs of primary intracerebral haemorrhage in computed tomograms of the brain. BMF 1987;279:379-81.

29 What pathological type of stroke is it? In: Warlow CP, Dennis MS, Van Gijn J, et al, Stroke. A practical guide to managenis MS, Van Gijn J, et al, Stroke. A practical
ment. Oxford: Blackwell, 1996;146-89.

30 Bogousslavsky J, Regli F, Uske A, et al. Early spontaneous haematoma in cerebral infarct. Neurology 1991;41:837-40.

1 Alberts MJ, Faulstich ME, Gray L. Stroke with negative brain magnetic resonance imaging. Stroke 1992;23:663-7.

32 What caused this transient or persisting ischaemic event? In: Warlow CP, Dennis MS, Van Gijn J, et al, Stroke. A practical guide to management. Oxford: Blackwell, 1996;190-257.

33 What caused this intracerebral haemorrhage? In: Warlow CP, Dennis MS, Van Gijn J, et al, Stroke. A practical guide to management. Oxford: Blackwell, 1996;287-321.

34 What are this person's problems? A problem-based approach to the general management of stroke. In: Warlow CP, Dennis MS, Van Gijn J, et al, Stroke. A practical guide to management. Oxford: Blackwell, 1996;477-544.

35 Rout MW, Lane DJ, Wollner L. Prognosis in acute cerebrovascular accidents in relation to respiratory pattern and blood gas tensions. BMF 1971;3:7-9.

36 Nachtmann A, Siebler M, Rose G, et al. Cheyne-Stokes respiration in ischemic stroke. Neurology 1995;45:820-1.

37 Carlsson A, Britton M. Blood pressure after stroke. A 1 year follow up study. Stroke 1993;24:195-9.

38 Wallace JD, Levy LL. Blood pressure after stroke. $\mathcal{F} A M A$ 1981;246:2177-80.

39 Blood Pressure in Acute Stroke Collaboration (BASC). Interventions for deliberately altering blood pressure in acute stroke (Cochrane Review). In: The Cochrane Library, Issue 4, 1999. Oxford: Update Software.

40 Powers WJ. Acute hypertension after stroke: the scientific basis for treatment decisions. Neurology 1993;43:461-7.

41 Bath FJ, Bath PMW. What is the correct management of blood pressure in acute stroke? The Blood Pressure in Acute Stroke Collaboration. Cerebrovasc Dis 1997;7:205-

42 Potter JF. What should we do about blood pressure and stroke? Q F Med 1999;92:63-6.

43 Britton M, de Faire U, Helmers C. Hazards of therapy for excessive hypertension in acute stroke. Acta Med Scand 1980;207:253-7.

44 Schwarz S, Schwab S, Bertram M, et al. Effects of hypertonic saline hydroxyethyl starch solution and manni-
tol in patients with increased intracranial pressure after stroke. Stroke 1998;29:1550-5.

45 Schwab S, Steiner T, Aschoff A, et al. Early hemicraniectomy in patients with complete middle cerebral artery infarction. Stroke 1998;29:1888-93.

46 Gautier JC. Stroke-in-progression. Stroke 1985;16:729-33.

47 Asplund K. Any progress on progressing stroke? Cerebrovasc Dis 1992;2:317-19.

48 Gordon C, Hewer RL, Wade DT. Dysphagia in acute stroke. BMF 1987;295:411-14

49 Axelsson K, Asplund K, Norberg A, et al. Eating problems and nutritional status during hospital stay of patients with severe stroke. Fournal of American Dietary Association 1989; 8:1092-6.

50 Davalos A, Ricart W, Gonzalex-Huix F, et al. Effect of malnutrition after acute stroke on clinical outcome. Stroke 1996;27:1028-32

51 Smithard DG, Renwick D, O'Neill PA. Change in nutritional stroke following acute stroke [abstract]. $A g e$ Ageing 1993;22(suppl 3):11.

52 Unosson M, Ek AC, Bjurulf P, et al. Feeding dependence and nutritional status after acute stroke. Stroke 1994;25: 366-71.

53 Smithard DG, O'Neill PA, Park C, et al. Complications and outcome after stroke. Does dysphagia matter? Stroke 1996; 27:1200-4.

54 Scottish intercollegiate guidelines network. Management of patients with stroke. III: Identification and management of dysphagia. Edinburgh: SIGN, 1997.

55 Smithard DG, O'Neill PA, Park C, et al. Can bedside assessment relaibly exclude aspiration following acute stroke? Age Ageing 1999;27:99-106.

56 Dennis MS. FOOD (feed or ordinary diet) a multicentre international randomised trial evaluating feeding policies for patients hospitalised with a recent stroke: introducing the concept of a family of trials [abstract]. Age Ageing 1998;27(suppl 1):68.

57 Scott JF, Gray CS, O'Connell JE, et al. Glucose and insulin therapy in acute stroke; why delay further? $Q \mathcal{F}$ Med 1998 ; 91:511-15.

58 Scott JS, Robinson GM, O'Connell JE, et al. Glucose potassium insulin (GKI) infusions in the treatment of acute stroke patients with mild to moderate hyperglycaemia: the glucose, insulin in stroke study (GIST). Stroke 2000; (in press).

59 Castillo J, Martinez F, Leira R, et al. Mortality and morbidity of acute cerebral infarction related to temperature and basal analytic parameters. Cerebrovasc Dis 1994;4:66-71.
60 Chen H, Chopp M, Welch KM. Effect of mild hyperthermia on the ischemic infarct volume after middle cerebral artery occlusion in the rat. Neurology 1991;41:1133-5.

61 Azzimondi G, Bassein L, Nonino F, et al. Fever in acute stroke worsens prognosis. A prospective study. Stroke 1995; 26:2040-3.

62 Reith J, Jorgensen HS, Pedersen PM, et al. Body temperature in acute stroke: relation to stroke severity, infarct size, mortality, and outcome. Lancet 1996;347:422-5.

63 Ginsberg MD, Busto R. Combating hyperthermia in acute stroke. A significant clinical concern. Stroke 1998;29:52934.

64 Schwab S, Schwarz S, Spranger M, et al. Moderate hypothermia in the treatment of patients with severe middle cerebral artery infarction. Stroke 1998;29:2461-6.

65 Nuffield Institute for Health. Effective health care. The prevention and treatment of pressure sores. Effective Health Care 1995;2:1-16.

66 Nakayama H, Jorgensen HS, Pedersen PM, et al. Prevalence and risk factors of incontinence after stroke. The Copenhagen stroke study. Stroke 1997;28:58-62.

67 Warlow C, Ogston D, Douglas AS. Venous thrombosis following strokes. Lancet 1972;i:1305-6.

68 Warlow C. Venous thromboembolism after stroke. Am Heart f 1978;96:283-5.

69 McClatchie G. Survey of the rehabilitation outcomes of stroke. Med F Aust 1980;1:649-51.

70 Wells PS, Lensing AWA, Hirsch J. Graduated pressure stockings in the prevention of postoperative veous thromboembolism: a meta analysis. Arch Intern Med 1994; 154:67-72.

71 Antiplatelet Trialists' Collaboration. Collaborative overview of randomised trials of antiplatelet therapy. III: Reduction in venous thrombosis and pulmonary embolism by antiplatelet prophylaxis among surgical and medical patients. $B M$ 1994:308:235-46.

72 Gubitz G, Counsell C, Sandercock P, et al. Anticoagulants in acute ischaemic stroke. (Cochrane Review). In: The Cochrane Library, Issue 4, 1999. Oxford: Update Software.

73 Kilpatrick CJ, Davis SM, Tress BM, et al. Epileptic seizures in acute stroke. Arch Neurol 1990;47:157-60.

74 So EL, Annegers JF, Hauser WA, et al. Population-based study of seizure disorders after cerebral infarction. Neurology 1996;46:350-5.

75 Burn J, Dennis M, Bamford J, et al. Epileptic seizures after a first stroke: the Oxfordshire community stroke project. BMF 1997;315:1582-7.

76 Scottish intercollegiate guidelines network. Diagnosis and management of epilepsy in adults. Edinburgh: SIGN, 1997.

77 Sharp FR, Swanson RA, Honkaniemi, et al. Neurochemistry and molecular biology. In: Barnett HJM, Mohr JP, Stein $\mathrm{BM}$, et al, eds. Stroke: pathophysiology, diagnosis, and management. Philadelphia: Churchill Livingstone, 1998.

78 Kristian T, Siesjo BK. Calcium in ischemic cell death. Stroke 1998;29:705-18.

79 Steiner T, Hacke W. Combination therapy with neuroprotectants and thrombolytics in acute ischaemic stroke. European Neurology 1998;40:1-8.

80 Marchal G, Beaudouin V, Rioux P, et al. Prolonged persistence of substantial volumes of potentially viable brain tissue after stroke: a correlative PET-CT study with voxelbased data analysis. Stroke 1996;27:599-606.

81 de Bruijn SF, Stam J. Randomized, placebo-controlled trial of antocoagulant treatment with low-molecular-weight heparin for cerebral sinus thrombosis. Stroke 1999;30:4848.

82 Wardlaw JM, Warlow CP, Counsell C. Systematic review of evidence on thrombolytic therapy for acute ischaemic stroke. Lancet 1997;350:607-14.

83 Wardlaw JM, Warlow CP. Thrombolytic therapy for acute ischaemic stroke: the updated Cochrane database of systematic reviews meta-analysis. Cerebrovasc Dis 2000; (in press).

84 Grond M, Stenzel C, Schmulling S, et al. Early intravenous thrombolysis for acute ischemic stroke in a communitybased approach. Stroke 1998;29:1544-9.

85 Koudstaal P. Anticoagulants for preventing stroke in patients with nonrheumatic atrial fibrillation and a history of stroke or transient ischemic attacks (Cochrane Review). In: The Cochrane Library, Issue 4, 1999. Oxford: Update Software.

86 Koudstaal P. Anticoagulants versus antiplatelet therapy for preventing stroke in patients with nonrheumatic atrial fibrillation and a history of stroke or transient ischemic attacks (Cochrane Review). In: The Cochrane Library, Issue 4, 1999. Oxford: Update Software.

87 CAST (Chinese acute stroke trial) Collaborative Group. CAST: randomised placebo-controlled trial of early aspirin use in 20000 patients with acute ischaemic stroke. Lancet use in 20000 patien

88 Lees KR, Muir KW. Excitatory amino acid modulators for acute stroke (Protocol for a Cochrane Review). In: The Cochrane Library, Issue 4, 1999. Oxford: Update Software.

89 Hankey GJ, Hon C. Surgery for primary intracerebral hemorrhage: is it safe and effective? A systematic review of case series and randomized trials. Stroke 1997;28:2126-32.

90 Gerritsen van der Hoop R, Vermeulen M, Van Gijn J. Cerebellar hemorrhage: diagnosis and treatment. Surg Neurol 1988;29:6-10. 
91 Bamford J, Sandercock P, Warlow C, et al. Why are patients with acute stroke admitted to hospital? BMF 1986;292 1369-72.

92 Wade DT, Hewer RL. Hospital admission for acute stroke: who, for how long, and to what effect? F Epidemiol Community Health 1985;39:347-52.

93 Brocklehurst JC, Andrews K, Morris P, et al. Why admit stroke patients to hospital? Age Ageing 1978;7:100-8.

94 Lanska DJ. Review criteria for hospital utilization for patients with cerebrovascular disease. Task Force on Hospital Utilization for Stroke of the American Academy of Neurology. Neurology 1994;44:1531-2.

95 Adams HP, Brott TG, Crowell RM, et al. Guidelines for the management of patients with acute ischaemic stroke. A statement for healthcare professionals from a special writing group of the Stroke Council, American Heart Association. Stroke 1994;25:1901-14.

96 Scottish Intercollegiate Guidelines Network. Management of patients with stroke. I: Assessment, investigation, management and secondary prevention. Edinburgh: SIGN, 1997.

97 Royal College of Physicians of Edinburgh. Consensus conference on the medical management of stroke. $f$ Neurol Neurosurg Psychiatry 1999;66:128-9.

98 Stroke Unit Trialists' Collaboration. Organised inpatient (stroke unit) care for stroke (Cochrane Review). In: The Cochrane Library, Issue 4, 1999. Oxford: Update Software.

99 Adams HP, Brott TG, Furlan AJ, et al. Guidelines for thrombolytic therapy for acute stroke: a supplement to the guidelines for the management of patients with acute ischemic stroke. Circulation 1996;94:1167-74. 